\title{
Solvability for $p$-Laplacian boundary value problem at resonance on the half-line
}

Weihua Jiang*

\section{"Correspondence:}

weihuajiang@hebust.edu.cn

College of Sciences, Hebei

University of Science and

Technology, Shijiazhuang, Hebei 050018, P.R. China

\begin{abstract}
The existence of solutions for $p$-Laplacian boundary value problem at resonance on the half-line is investigated. Our analysis relies on constructing the suitable Banach space, defining appropriate operators and using the extension of Mawhin's continuation theorem. An example is given to illustrate our main result.
\end{abstract}

MSC: 70K30; 34B10; 34B15

Keywords: $p$-Laplacian; resonance; half-line; multi-point boundary value problem; continuation theorem

\section{Introduction}

A boundary value problem is said to be a resonance one if the corresponding homogeneous boundary value problem has a non-trivial solution. Resonance problems can be expressed as an abstract equation $L x=N x$, where $L$ is a noninvertible operator. When $L$ is linear, Mawhin's continuation theorem [1] is an effective tool in finding solutions for these problems, see [2-10] and references cited therein. But it does not work when $L$ is nonlinear, for instance, $p$-Laplacian operator. In order to solve this problem, Ge and Ren [11] proved a continuation theorem for the abstract equation $L x=N x$ when $L$ is a noninvertible nonlinear operator and used it to study the existence of solutions for the boundary value problems with a $p$-Laplacian:

$$
\left\{\begin{array}{l}
\left(\varphi_{p}\left(u^{\prime}\right)\right)^{\prime}+f(t, u)=0, \quad 0<t<1, \\
u(0)=0=G(u(\eta), u(1)),
\end{array}\right.
$$

where $\varphi_{p}(s)=|s|^{p-2} s, p>1,0<\eta<1 . \varphi_{p}(s)$ is nonlinear when $p \neq 2$.

As far as the boundary value problems on unbounded domain are concerned, there are many excellent results, see [12-15] and references cited therein.

To the best of our knowledge, there are few papers that study the $p$-Laplacian boundary value problem at resonance on the half-line. In this paper, we investigate the existence of solutions for the boundary value problem

$$
\left\{\begin{array}{l}
\left(\varphi_{p}\left(u^{\prime}\right)\right)^{\prime}+f\left(t, u, u^{\prime}\right)=0, \quad 0<t<+\infty, \\
u(0)=0, \quad \varphi_{p}\left(u^{\prime}(+\infty)\right)=\sum_{i=1}^{n} \alpha_{i} \varphi_{p}\left(u^{\prime}\left(\xi_{i}\right)\right),
\end{array}\right.
$$

where $\alpha_{i}>0, i=1,2, \ldots, n, \sum_{i=1}^{n} \alpha_{i}=1$. 
In order to obtain our main results, we always suppose that the following conditions hold.

$\left(\mathrm{H}_{1}\right) \quad 0<\xi_{1}<\xi_{2}<\cdots<\xi_{n}<+\infty, \alpha_{i}>0, \sum_{i=1}^{n} \alpha_{i}=1$.

$\left(\mathrm{H}_{2}\right) f:[0,+\infty) \times \mathbb{R}^{2} \rightarrow \mathbb{R}$ is continuous, $f(t, 0,0) \neq 0, t \in(0, \infty)$ and for any $r>0$, there exists a nonnegative function $h_{r}(t) \in L^{1}[0,+\infty)$ such that

$$
|f(t, x, y)| \leq h_{r}(t), \quad \text { a.e. } t \in[0,+\infty), x, y \in \mathbb{R}, \frac{|x|}{1+t} \leq r,|y| \leq r .
$$

\section{Preliminaries}

For convenience, we introduce some notations and a theorem. For more details, see [11].

Definition 2.1 [11] Let $X$ and $Y$ be two Banach spaces with the norms $\|\cdot\|_{X},\|\cdot\|_{Y}$, respectively. A continuous operator $M: X \cap \operatorname{dom} M \rightarrow Y$ is said to be quasi-linear if

(i) $\operatorname{Im} M:=M(X \cap \operatorname{dom} M)$ is a closed subset of $Y$,

(ii) $\operatorname{Ker} M:=\{x \in X \cap \operatorname{dom} M: M x=0\}$ is linearly homeomorphic to $\mathbb{R}^{n}, n<\infty$, where $\operatorname{dom} M$ denote the domain of the operator $M$.

Let $X_{1}=\operatorname{Ker} M$ and $X_{2}$ be the complement space of $X_{1}$ in $X$, then $X=X_{1} \oplus X_{2}$. On the other hand, suppose that $Y_{1}$ is a subspace of $Y$, and that $Y_{2}$ is the complement of $Y_{1}$ in $Y$, i.e., $Y=Y_{1} \oplus Y_{2}$. Let $P: X \rightarrow X_{1}$ and $Q: Y \rightarrow Y_{1}$ be two projectors and $\Omega \subset X$ an open and bounded set with the origin $\theta \in \Omega$.

Definition 2.2 [11] Suppose that $N_{\lambda}: \bar{\Omega} \rightarrow Y, \lambda \in[0,1]$ is a continuous operator. Denote $N_{1}$ by $N$. Let $\Sigma_{\lambda}=\left\{x \in \bar{\Omega}: M x=N_{\lambda} x\right\} . N_{\lambda}$ is said to be $M$-compact in $\bar{\Omega}$ if there exist a vector subspace $Y_{1}$ of $Y$ satisfying $\operatorname{dim} Y_{1}=\operatorname{dim} X_{1}$ and an operator $R: \bar{\Omega} \times[0,1] \rightarrow X_{2}$ being continuous and compact such that for $\lambda \in[0,1]$,

(a) $(I-Q) N_{\lambda}(\bar{\Omega}) \subset \operatorname{Im} M \subset(I-Q) Y$,

(b) $Q N_{\lambda} x=\theta, \lambda \in(0,1) \Leftrightarrow Q N x=\theta$,

(c) $R(\cdot, 0)$ is the zero operator and $\left.R(\cdot, \lambda)\right|_{\Sigma_{\lambda}}=\left.(I-P)\right|_{\Sigma_{\lambda}}$,

(d) $M[P+R(\cdot, \lambda)]=(I-Q) N_{\lambda}$.

Theorem 2.1 [11] Let $X$ and $Y$ be two Banach spaces with the norms $\|\cdot\|_{X},\|\cdot\|_{Y}$, respectively, and $\Omega \subset X$ an open and bounded nonempty set. Suppose that

$$
M: X \cap \operatorname{dom} M \rightarrow Y
$$

is a quasi-linear operator and $N_{\lambda}: \bar{\Omega} \rightarrow Y, \lambda \in[0,1] M$-compact. In addition, if the following conditions hold:

$\left(C_{1}\right) M x \neq N_{\lambda} x, \forall x \in \partial \Omega \cap \operatorname{dom} M, \lambda \in(0,1)$,

$\left(C_{2}\right) \operatorname{deg}\{J Q N, \Omega \cap \operatorname{Ker} M, 0\} \neq 0$,

then the abstract equation $M x=N x$ has at least one solution in $\operatorname{dom} M \cap \bar{\Omega}$, where $N=N_{1}$, $J: \operatorname{Im} Q \rightarrow \operatorname{Ker} M$ is a homeomorphism with $J(\theta)=\theta$. 


\section{Main result}

Let $X=\left\{u \mid u \in C^{1}[0,+\infty), u(0)=0, \sup _{t \in[0,+\infty)} \frac{|u(t)|}{1+t}<+\infty, \lim _{t \rightarrow+\infty} u^{\prime}(t)\right.$ exists $\}$ with norm $\|u\|=\max \left\{\left\|\frac{u}{1+t}\right\|_{\infty},\left\|u^{\prime}\right\|_{\infty}\right\}$, where $\|u\|_{\infty}=\sup _{t \in[0,+\infty)}|u(t)| . \quad Y=L^{1}[0,+\infty)$ with norm $\|y\|_{1}=\int_{0}^{+\infty}|y(t)| d t$. Then $(X,\|\cdot\|)$ and $\left(Y,\|\cdot\|_{1}\right)$ are Banach spaces.

Define operators $M: X \cap \operatorname{dom} M \rightarrow Y$ and $N_{\lambda}: X \rightarrow Y$ as follows:

$$
M u=\left(\varphi_{p}\left(u^{\prime}\right)\right)^{\prime}, \quad N_{\lambda} u=-\lambda f\left(t, u, u^{\prime}\right), \quad \lambda \in[0,1], t \in[0,+\infty),
$$

where

$$
\begin{aligned}
\operatorname{dom} M= & \left\{u \in X \mid \varphi_{p}\left(u^{\prime}\right) \in A C[0,+\infty),\left(\varphi_{p}\left(u^{\prime}\right)\right)^{\prime} \in L^{1}[0,+\infty),\right. \\
& \left.\varphi_{p}\left(u^{\prime}(+\infty)\right)=\sum_{i=1}^{n} \alpha_{i} \varphi_{p}\left(u^{\prime}\left(\xi_{i}\right)\right)\right\}
\end{aligned}
$$

Then the boundary value problem (1.1) is equivalent to $M u=N u$.

Obviously,

$$
\operatorname{Ker} M=\{a t \mid a \in \mathbb{R}\}, \quad \operatorname{Im} M=\left\{y \mid y \in Y, \sum_{i=1}^{n} \alpha_{i} \int_{\xi_{i}}^{+\infty} y(s) d s=0\right\} .
$$

It is clear that $\operatorname{Ker} M$ is linearly homeomorphic to $\mathbb{R}$, and $\operatorname{Im} M \subset Y$ is closed. So, $M$ is a quasi-linear operator.

Define $P: X \rightarrow X_{1}, Q: Y \rightarrow Y_{1}$ as

$$
(P u)(t)=u^{\prime}(+\infty) t, \quad(Q y)(t)=\frac{\sum_{i=1}^{n} \alpha_{i} \int_{\xi_{i}}^{+\infty} y(s) d s}{\sum_{i=1}^{n} \alpha_{i} e^{-\xi_{i}}} e^{-t},
$$

where $X_{1}=\operatorname{Ker} M, Y_{1}=\operatorname{Im} Q=\left\{b e^{-t} \mid b \in \mathbb{R}\right\}$. We can easily obtain that $P: X \rightarrow X_{1}, Q: Y \rightarrow$ $Y_{1}$ are projectors. Set $X=X_{1} \oplus X_{2}, Y=Y_{1} \oplus Y_{2}$.

Define an operator $R: X \times[0,1] \rightarrow X_{2}$ :

$$
\begin{aligned}
R(u, \lambda)(t)= & \int_{0}^{t} \varphi_{q}\left[\int_{\tau}^{+\infty} \lambda\left(f\left(s, u(s), u^{\prime}(s)\right)-\frac{\sum_{i=1}^{n} \alpha_{i} \int_{\xi_{i}}^{+\infty} f\left(r, u(r), u^{\prime}(r)\right) d r}{\sum_{i=1}^{n} \alpha_{i} e^{-\xi_{i}}} e^{-s}\right) d s\right. \\
& \left.+\varphi_{p}\left(u^{\prime}(+\infty)\right)\right] d \tau-u^{\prime}(+\infty) t,
\end{aligned}
$$

where $\frac{1}{p}+\frac{1}{q}=1, \varphi_{q}=\varphi_{p}^{-1}$. By $\left(\mathrm{H}_{1}\right)$ and $\left(\mathrm{H}_{2}\right)$, we get that $R: X \times[0,1] \rightarrow X_{2}$ is continuous.

Lemma 3.1 [15] $V \subset X$ is compact if $\left\{\frac{u(t)}{1+t} \mid u \in V\right\}$ and $\left\{u^{\prime}(t) \mid u \in V\right\}$ are both equicontinuous on any compact intervals of $[0,+\infty)$ and equiconvergent at infinity.

Lemma 3.2 $R: X \times[0,1] \rightarrow X_{2}$ is compact.

Proof Let $\Omega \subset X$ be nonempty and bounded. There exists a constant $r>0$ such that $\|u\| \leq$ $r, u \in \bar{\Omega}$. It follows from $\left(\mathrm{H}_{2}\right)$ that there exists a nonnegative function $h_{r}(t) \in L^{1}[0,+\infty)$ 
such that

$$
\left|f\left(t, u(t), u^{\prime}(t)\right)\right| \leq h_{r}(t), \quad \text { a.e. } t \in[0,+\infty), u \in \bar{\Omega} .
$$

For any $T>0, t_{1}, t_{2} \in[0, T], u \in \bar{\Omega}, \lambda \in[0,1]$, we have

$$
\begin{aligned}
& \left|\frac{R(u, \lambda)\left(t_{1}\right)}{1+t_{1}}-\frac{R(u, \lambda)\left(t_{2}\right)}{1+t_{2}}\right| \\
& \leq \mid \frac{1}{1+t_{1}} \int_{0}^{t_{1}} \varphi_{q}\left[\int_{\tau}^{+\infty} \lambda\left(f\left(s, u(s), u^{\prime}(s)\right)-\frac{\sum_{i=1}^{n} \alpha_{i} \int_{\xi_{i}}^{+\infty} f\left(r, u(r), u^{\prime}(r)\right) d r}{\sum_{i=1}^{n} \alpha_{i} e^{-\xi_{i}}} e^{-s}\right) d s\right. \\
& \left.+\varphi_{p}\left(u^{\prime}(+\infty)\right)\right] d \tau \\
& -\frac{1}{1+t_{2}} \int_{0}^{t_{2}} \varphi_{q}\left[\int_{\tau}^{+\infty} \lambda\left(f\left(s, u(s), u^{\prime}(s)\right)-\frac{\sum_{i=1}^{n} \alpha_{i} \int_{\xi_{i}}^{+\infty} f\left(r, u(r), u^{\prime}(r)\right) d r}{\sum_{i=1}^{n} \alpha_{i} e^{-\xi_{i}}} e^{-s}\right) d s\right. \\
& \left.+\varphi_{p}\left(u^{\prime}(+\infty)\right)\right] d \tau|+| \frac{t_{1}}{1+t_{1}}-\frac{t_{2}}{1+t_{2}}|| u^{\prime}(+\infty) \mid \\
& \leq \mid \frac{1}{1+t_{1}} \int_{t_{2}}^{t_{1}} \varphi_{q}\left[\int_{\tau}^{+\infty} \lambda\left(f\left(s, u(s), u^{\prime}(s)\right)-\frac{\sum_{i=1}^{n} \alpha_{i} \int_{\xi_{i}}^{+\infty} f\left(r, u(r), u^{\prime}(r)\right) d r}{\sum_{i=1}^{n} \alpha_{i} e^{-\xi_{i}}} e^{-s}\right) d s\right. \\
& \left.+\varphi_{p}\left(u^{\prime}(+\infty)\right)\right] d \tau|+| \frac{1}{1+t_{1}}-\frac{1}{1+t_{2}} \mid \\
& \times \mid \int_{0}^{t_{2}} \varphi_{q}\left[\int_{\tau}^{+\infty} \lambda\left(f\left(s, u(s), u^{\prime}(s)\right)-\frac{\sum_{i=1}^{n} \alpha_{i} \int_{\xi_{i}}^{+\infty} f\left(r, u(r), u^{\prime}(r)\right) d r}{\sum_{i=1}^{n} \alpha_{i} e^{-\xi_{i}}} e^{-s}\right) d s\right. \\
& \left.+\varphi_{p}\left(u^{\prime}(+\infty)\right)\right] d \tau|+| \frac{t_{1}}{1+t_{1}}-\frac{t_{2}}{1+t_{2}} \mid r \\
& \leq \varphi_{q}\left[\left\|h_{r}\right\|_{1}\left(1+\frac{1}{\sum_{i=1}^{n} \alpha_{i} e^{-\xi_{i}}}\right)+\varphi_{p}(r)\right]\left[\left|t_{1}-t_{2}\right|+T\left|\frac{1}{1+t_{1}}-\frac{1}{1+t_{2}}\right|\right] \\
& +\left|\frac{t_{1}}{1+t_{1}}-\frac{t_{2}}{1+t_{2}}\right| r \text {. }
\end{aligned}
$$

Since $\left\{t, \frac{1}{1+t}, \frac{t}{1+t}\right\}$ are equicontinuous on $[0, T]$, we get that $\left\{\frac{R(u, \lambda)(t)}{1+t}, u \in \bar{\Omega}\right\}$ are equicontinuous on $[0, T]$.

$$
\begin{aligned}
& \left|R(u, \lambda)^{\prime}\left(t_{1}\right)-R(u, \lambda)^{\prime}\left(t_{2}\right)\right| \\
& =\mid \varphi_{q}\left[\int_{t_{1}}^{+\infty} \lambda\left(f\left(s, u(s), u^{\prime}(s)\right)-\frac{\sum_{i=1}^{n} \alpha_{i} \int_{\xi_{i}}^{+\infty} f\left(r, u(r), u^{\prime}(r)\right) d r}{\sum_{i=1}^{n} \alpha_{i} e^{-\xi_{i}}} e^{-s}\right) d s\right. \\
& \left.\quad+\varphi_{p}\left(u^{\prime}(+\infty)\right)\right] \\
& \quad-\varphi_{q}\left[\int_{t_{2}}^{+\infty} \lambda\left(f\left(s, u(s), u^{\prime}(s)\right)-\frac{\sum_{i=1}^{n} \alpha_{i} \int_{\xi_{i}}^{+\infty} f\left(r, u(r), u^{\prime}(r)\right) d r}{\sum_{i=1}^{n} \alpha_{i} e^{-\xi_{i}}} e^{-s}\right) d s\right. \\
& \left.\quad+\varphi_{p}\left(u^{\prime}(+\infty)\right)\right] .
\end{aligned}
$$


Let

$$
g(t, u)=\int_{t}^{+\infty} \lambda\left(f\left(s, u(s), u^{\prime}(s)\right)-\frac{\sum_{i=1}^{n} \alpha_{i} \int_{\xi_{i}}^{+\infty} f\left(r, u(r), u^{\prime}(r)\right) d r}{\sum_{i=1}^{n} \alpha_{i} e^{-\xi_{i}}} e^{-s}\right) d s+\varphi_{p}\left(u^{\prime}(+\infty)\right) .
$$

Then

$$
|g(t, u)| \leq\left\|h_{r}\right\|_{1}\left(1+\frac{1}{\sum_{i=1}^{n} \alpha_{i} e^{-\xi_{i}}}\right)+\varphi_{p}(r):=k, \quad t \in[0, T], u \in \bar{\Omega} .
$$

For $t_{1}, t_{2} \in[0, T], t_{1}<t_{2}, u \in \bar{\Omega}$, we have

$$
\begin{aligned}
\left|g\left(t_{1}, u\right)-g\left(t_{2}, u\right)\right| & =\left|\int_{t_{1}}^{t_{2}} \lambda\left(f\left(s, u(s), u^{\prime}(s)\right)-\frac{\sum_{i=1}^{n} \alpha_{i} \int_{\xi_{i}}^{+\infty} f\left(r, u(r), u^{\prime}(r)\right) d r}{\sum_{i=1}^{n} \alpha_{i} e^{-\xi_{i}}} e^{-s}\right) d s\right| \\
& \leq \int_{t_{1}}^{t_{2}} h_{r}(s)+\frac{\left\|h_{r}\right\|_{1}}{\sum_{i=1}^{n} \alpha_{i} e^{-\xi_{i}}} e^{-s} d s .
\end{aligned}
$$

It follows from the absolute continuity of integral that $\{g(t, u), u \in \bar{\Omega}\}$ are equicontinuous on $[0, T]$. Since $\varphi_{q}(x)$ is uniformly continuous on $[-k, k]$, by $(3.1)$, we can obtain that $\left\{R(u, \lambda)^{\prime}(t), u \in \bar{\Omega}\right\}$ are equicontinuous on $[0, T]$.

For $u \in \bar{\Omega}$, since

$$
\begin{aligned}
& \left|\int_{\tau}^{+\infty} \lambda\left(f\left(s, u(s), u^{\prime}(s)\right)-\frac{\sum_{i=1}^{n} \alpha_{i} \int_{\xi_{i}}^{+\infty} f\left(r, u(r), u^{\prime}(r)\right) d r}{\sum_{i=1}^{n} \alpha_{i} e^{-\xi_{i}}} e^{-s}\right) d s\right| \\
& \quad \leq \int_{\tau}^{+\infty} h_{r}(s)+\frac{\left\|h_{r}\right\|_{1}}{\sum_{i=1}^{n} \alpha_{i} e^{-\xi_{i}}} e^{-s} d s, \\
& \quad \lim _{\tau \rightarrow+\infty} \int_{\tau}^{+\infty} h_{r}(s)+\frac{\left\|h_{r}\right\|_{1}}{\sum_{i=1}^{n} \alpha_{i} e^{-\xi_{i}}} e^{-s} d s=0,
\end{aligned}
$$

and $\varphi_{q}(x)$ is uniformly continuous on $\left[-r-r^{p-1}, r+r^{p-1}\right]$, for any $\varepsilon>0$, there exists a constant $T_{1}>0$ such that if $\tau \geq T_{1}$, then

$$
\begin{aligned}
\mid \varphi_{q} & {\left[\int_{\tau}^{+\infty} \lambda\left(f\left(s, u(s), u^{\prime}(s)\right)-\frac{\sum_{i=1}^{n} \alpha_{i} \int_{\xi_{i}}^{+\infty} f\left(r, u(r), u^{\prime}(r)\right) d r}{\sum_{i=1}^{n} \alpha_{i} e^{-\xi_{i}}} e^{-s}\right) d s+\varphi_{p}\left(u^{\prime}(+\infty)\right)\right] } \\
& -u^{\prime}(+\infty) \mid<\frac{\varepsilon}{4}, \quad \forall u \in \bar{\Omega} .
\end{aligned}
$$

Since

$$
\begin{aligned}
& \mid \int_{0}^{T_{1}} \varphi_{q}\left[\int_{\tau}^{+\infty} \lambda\left(f\left(s, u(s), u^{\prime}(s)\right)-\frac{\sum_{i=1}^{n} \alpha_{i} \int_{\xi_{i}}^{+\infty} f\left(r, u(r), u^{\prime}(r)\right) d r}{\sum_{i=1}^{n} \alpha_{i} e^{-\xi_{i}}} e^{-s}\right) d s\right. \\
& \left.\quad+\varphi_{p}\left(u^{\prime}(+\infty)\right)\right] d \tau-u^{\prime}(+\infty) T_{1} \mid \\
& \quad \leq\left\{\varphi_{q}\left[\left\|h_{r}\right\|_{1}\left(1+\frac{1}{\sum_{i=1}^{n} \alpha_{i} e^{-\xi_{i}}}\right)+\varphi_{p}(r)\right]+r\right\} T_{1},
\end{aligned}
$$


there exists a constant $T>T_{1}$ such that if $t>T$, then

$$
\frac{1}{1+t}\left\{\varphi_{q}\left[\left\|h_{r}\right\|_{1}\left(1+\frac{1}{\sum_{i=1}^{n} \alpha_{i} e^{-\xi_{i}}}\right)+\varphi_{p}(r)\right]+r\right\} T_{1}<\frac{\varepsilon}{4}
$$

For $t_{2}>t_{1}>T$, by (3.2), (3.3) and (3.4), we have

$$
\begin{aligned}
& \left|\frac{R(u, \lambda)\left(t_{1}\right)}{1+t_{1}}-\frac{R(u, \lambda)\left(t_{2}\right)}{1+t_{2}}\right| \\
& =\mid \frac{1}{1+t_{1}} \int_{0}^{t_{1}}\left\{\varphi _ { q } \left[\int_{\tau}^{+\infty} \lambda\left(f\left(s, u(s), u^{\prime}(s)\right)-\frac{\sum_{i=1}^{n} \alpha_{i} \int_{\xi_{i}}^{+\infty} f\left(r, u(r), u^{\prime}(r)\right) d r}{\sum_{i=1}^{n} \alpha_{i} e^{-\xi_{i}}} e^{-s}\right) d s\right.\right. \\
& \left.\left.+\varphi_{p}\left(u^{\prime}(+\infty)\right)\right]-u^{\prime}(+\infty)\right\} d \tau \\
& -\frac{1}{1+t_{2}} \int_{0}^{t_{2}}\left\{\varphi _ { q } \left[\int_{\tau}^{+\infty} \lambda\left(f\left(s, u(s), u^{\prime}(s)\right)-\frac{\sum_{i=1}^{n} \alpha_{i} \int_{\xi_{i}}^{+\infty} f\left(r, u(r), u^{\prime}(r)\right) d r}{\sum_{i=1}^{n} \alpha_{i} e^{-\xi_{i}}} e^{-s}\right) d s\right.\right. \\
& \left.\left.+\varphi_{p}\left(u^{\prime}(+\infty)\right)\right]-u^{\prime}(+\infty)\right\} d \tau \\
& \leq \mid \frac{1}{1+t_{1}} \int_{0}^{T_{1}}\left\{\varphi _ { q } \left[\int_{\tau}^{+\infty} \lambda\left(f\left(s, u(s), u^{\prime}(s)\right)-\frac{\sum_{i=1}^{n} \alpha_{i} \int_{\xi_{i}}^{+\infty} f\left(r, u(r), u^{\prime}(r)\right) d r}{\sum_{i=1}^{n} \alpha_{i} e^{-\xi_{i}}} e^{-s}\right) d s\right.\right. \\
& \left.\left.+\varphi_{p}\left(u^{\prime}(+\infty)\right)\right]-u^{\prime}(+\infty)\right\} d \tau \mid \\
& +\mid \frac{1}{1+t_{1}} \int_{T_{1}}^{t_{1}}\left\{\varphi _ { q } \left[\int_{\tau}^{+\infty} \lambda\left(f\left(s, u(s), u^{\prime}(s)\right)-\frac{\sum_{i=1}^{n} \alpha_{i} \int_{\xi_{i}}^{+\infty} f\left(r, u(r), u^{\prime}(r)\right) d r}{\sum_{i=1}^{n} \alpha_{i} e^{-\xi_{i}}} e^{-s}\right) d s\right.\right. \\
& \left.\left.+\varphi_{p}\left(u^{\prime}(+\infty)\right)\right]-u^{\prime}(+\infty)\right\} d \tau \mid \\
& +\mid \frac{1}{1+t_{2}} \int_{0}^{T_{1}}\left\{\varphi _ { q } \left[\int_{\tau}^{+\infty} \lambda\left(f\left(s, u(s), u^{\prime}(s)\right)-\frac{\sum_{i=1}^{n} \alpha_{i} \int_{\xi_{i}}^{+\infty} f\left(r, u(r), u^{\prime}(r)\right) d r}{\sum_{i=1}^{n} \alpha_{i} e^{-\xi_{i}}} e^{-s}\right) d s\right.\right. \\
& \left.\left.+\varphi_{p}\left(u^{\prime}(+\infty)\right)\right]-u^{\prime}(+\infty)\right\} d \tau \\
& +\mid \frac{1}{1+t_{2}} \int_{T_{1}}^{t_{2}}\left\{\varphi _ { q } \left[\int_{\tau}^{+\infty} \lambda\left(f\left(s, u(s), u^{\prime}(s)\right)-\frac{\sum_{i=1}^{n} \alpha_{i} \int_{\xi_{i}}^{+\infty} f\left(r, u(r), u^{\prime}(r)\right) d r}{\sum_{i=1}^{n} \alpha_{i} e^{-\xi_{i}}} e^{-s}\right) d s\right.\right. \\
& \left.\left.+\varphi_{p}\left(u^{\prime}(+\infty)\right)\right]-u^{\prime}(+\infty)\right\} d \tau \mid<\varepsilon
\end{aligned}
$$

and

$$
\begin{aligned}
& \left|R(u, \lambda)^{\prime}\left(t_{1}\right)-R(u, \lambda)^{\prime}\left(t_{2}\right)\right| \\
& \leq \mid \varphi_{q}\left[\int_{t_{1}}^{+\infty} \lambda\left(f\left(s, u(s), u^{\prime}(s)\right)-\frac{\sum_{i=1}^{n} \alpha_{i} \int_{\xi_{i}}^{+\infty} f\left(r, u(r), u^{\prime}(r)\right) d r}{\sum_{i=1}^{n} \alpha_{i} e^{-\xi_{i}}} e^{-s}\right) d s\right. \\
& \left.\quad+\varphi_{p}\left(u^{\prime}(+\infty)\right)\right]-u^{\prime}(+\infty) \mid \\
& \quad+\mid \varphi_{q}\left[\int_{t_{2}}^{+\infty} \lambda\left(f\left(s, u(s), u^{\prime}(s)\right)-\frac{\sum_{i=1}^{n} \alpha_{i} \int_{\xi_{i}}^{+\infty} f\left(r, u(r), u^{\prime}(r)\right) d r}{\sum_{i=1}^{n} \alpha_{i} e^{-\xi_{i}}} e^{-s}\right) d s\right.
\end{aligned}
$$




$$
\left.+\varphi_{p}\left(u^{\prime}(+\infty)\right)\right]-u^{\prime}(+\infty)
$$

$<\varepsilon$.

By Lemma 3.1, we get that $\{R(u, \lambda) \mid u \in \bar{\Omega}, \lambda \in[0,1]\}$ is compact. The proof is completed.

In the spaces $X$ and $Y$, the origin $\theta=0$. In the following sections, we denote the origin by 0 .

Lemma 3.3 Let $\Omega \subset X$ be nonempty, open and bounded. Then $N_{\lambda}$ is $M$-compact in $\bar{\Omega}$.

Proof By $\left(\mathrm{H}_{2}\right)$, we know that $N_{\lambda}: \bar{\Omega} \rightarrow Y$ is continuous. Obviously, $\operatorname{dim} X_{1}=\operatorname{dim} Y_{1}$. For $u \in \bar{\Omega}$, since $Q(I-Q)$ is a zero operator, we get $(I-Q) N_{\lambda}(u) \in \operatorname{Im} M$. For $y \in \operatorname{Im} M, y=$ $Q y+(I-Q) y=(I-Q) y \in(I-Q) Y$. So, we have $(I-Q) N_{\lambda}(\bar{\Omega}) \subset \operatorname{Im} M \subset(I-Q) Y$. It is clear that

$$
Q N_{\lambda} u=0, \quad \lambda \in(0,1) \quad \Leftrightarrow \quad Q N u=0
$$

and $R(u, 0)=0, \forall u \in X . u \in \Sigma_{\lambda}=\left\{u \in \bar{\Omega}: M u=N_{\lambda} u\right\}$ means that $N_{\lambda} u \in \operatorname{Im} M$ and $\left(\varphi_{p}\left(u^{\prime}\right)\right)^{\prime}+\lambda f\left(t, u, u^{\prime}\right)=0$, thus,

$$
\begin{aligned}
R(u, \lambda)(t) & =\int_{0}^{t} \varphi_{q}\left[\int_{\tau}^{+\infty}-\left(\varphi_{p}\left(u^{\prime}\right)\right)^{\prime} d s+\varphi_{p}\left(u^{\prime}(+\infty)\right)\right] d \tau-u^{\prime}(+\infty) t \\
& =u(t)-u^{\prime}(+\infty) t=(I-P) u(t) .
\end{aligned}
$$

For $u \in X$, we have

$$
\begin{aligned}
M[P+R(u, \lambda)](t) & =-\lambda f\left(t, u(t), u^{\prime}(t)\right)+\frac{\sum_{i=1}^{n} \alpha_{i} \int_{\xi_{i}}^{+\infty} \lambda f\left(r, u(r), u^{\prime}(r)\right) d r}{\sum_{i=1}^{n} \alpha_{i} e^{-\xi_{i}}} e^{-t} \\
& =(I-Q) N_{\lambda} u(t) .
\end{aligned}
$$

These, together with Lemma 3.2, mean that $N_{\lambda}$ is $M$-compact in $\bar{\Omega}$. The proof is completed.

In order to obtain our main results, we need the following additional conditions.

$\left(\mathrm{H}_{3}\right)$ There exist nonnegative functions $a(t), b(t), c(t)$ with $(1+t)^{p-1} a(t), b(t), c(t) \in Y$ and $\left\|(1+t)^{p-1} a(t)\right\|_{1}+\|b(t)\|_{1}<1$ such that

$$
|f(t, x, y)| \leq a(t)\left|\varphi_{p}(x)\right|+b(t)\left|\varphi_{p}(y)\right|+c(t), \quad \text { a.e. } t \in[0,+\infty)
$$

$\left(\mathrm{H}_{4}\right)$ There exists a constant $d_{0}>0$ such that if $|d|>d_{0}$, then one of the following inequalities holds:

$$
\begin{array}{ll}
d f(t, x, d)<0, & (t, x) \in[0,+\infty) \times \mathbb{R} \\
d f(t, x, d)>0, & (t, x) \in[0,+\infty) \times \mathbb{R} .
\end{array}
$$


Lemma 3.4 Assume that $\left(\mathrm{H}_{3}\right)$ and $\left(\mathrm{H}_{4}\right)$ hold. The set

$$
\Omega_{1}=\left\{u \mid u \in \operatorname{dom} M, M u=N_{\lambda} u, \lambda \in[0,1]\right\}
$$

is bounded in $X$.

Proof If $u \in \Omega_{1}$, then $Q N_{\lambda} u=0$, i.e., $\sum_{i=1}^{n} \alpha_{i} \int_{\xi_{i}}^{+\infty} f\left(r, u(r), u^{\prime}(r)\right) d r=0$. By $\left(\mathrm{H}_{4}\right)$, there exists $t_{0} \in[0,+\infty)$ such that $\left|u^{\prime}\left(t_{0}\right)\right| \leq d_{0}$. It follows from $M u=N_{\lambda} u$ that

$$
\varphi_{p}\left(u^{\prime}(t)\right)=-\int_{t_{0}}^{t} \lambda f\left(s, u(s), u^{\prime}(s)\right) d s+\varphi_{p}\left(u^{\prime}\left(t_{0}\right)\right)
$$

Considering $\left(\mathrm{H}_{3}\right)$, we have

$$
\begin{aligned}
\left|\varphi_{p}\left(u^{\prime}(t)\right)\right| & \leq \int_{0}^{+\infty}\left[a(t)\left|\varphi_{p}(u(t))\right|+b(t)\left|\varphi_{p}\left(u^{\prime}(t)\right)\right|+c(t)\right] d t+\varphi_{p}\left(d_{0}\right) \\
& \leq\left\|a(t)(1+t)^{p-1}\right\|_{1} \varphi_{p}\left(\left\|\frac{u}{1+t}\right\|_{\infty}\right)+\|b\|_{1} \varphi_{p}\left(\left\|u^{\prime}\right\|_{\infty}\right)+\|c\|_{1}+\varphi_{p}\left(d_{0}\right) .
\end{aligned}
$$

Since $u(t)=\int_{0}^{t} u^{\prime}(s) d s$, we get

$$
\left|\frac{u(t)}{1+t}\right| \leq \frac{t}{1+t}\left\|u^{\prime}\right\|_{\infty} \leq\left\|u^{\prime}\right\|_{\infty} .
$$

Thus,

$$
\left\|\frac{u}{1+t}\right\|_{\infty} \leq\left\|u^{\prime}\right\|_{\infty}
$$

By (3.5), (3.6) and $\left(\mathrm{H}_{3}\right)$, we get

$$
\left\|\varphi_{p}\left(u^{\prime}\right)\right\|_{\infty} \leq \frac{\|c\|_{1}+\varphi_{p}\left(d_{0}\right)}{1-\left\|a(t)(1+t)^{p-1}\right\|_{1}-\|b\|_{1}} .
$$

So,

$$
\left\|u^{\prime}\right\|_{\infty} \leq \varphi_{q}\left(\frac{\|c\|_{1}+\varphi_{p}\left(d_{0}\right)}{1-\left\|a(t)(1+t)^{p-1}\right\|_{1}-\|b\|_{1}}\right) .
$$

This, together with (3.6), means that $\Omega_{1}$ is bounded. The proof is completed.

Lemma 3.5 Assume that $\left(\mathrm{H}_{4}\right)$ holds. The set

$$
\Omega_{2}=\{u \mid u \in \operatorname{Ker} M, Q N u=0\}
$$

is bounded in $X$.

Proof $u \in \Omega_{2}$ means that $u=a t, a \in \mathbb{R}$ and $Q N u=0$, i.e.,

$$
\sum_{i=1}^{n} \alpha_{i} \int_{\xi_{i}}^{+\infty} f(s, a s, a) d s=0 .
$$

By $\left(\mathrm{H}_{4}\right)$, we get that $|a| \leq d_{0}$. So, $\Omega_{2}$ is bounded. The proof is completed. 
Theorem 3.1 Suppose that $\left(\mathrm{H}_{1}\right)-\left(\mathrm{H}_{4}\right)$ hold. Then problem (1.1) has at least one solution.

Proof Let $\Omega=\left\{u \in X \mid\|u\|<d_{0}^{\prime}\right\}$, where $d_{0}^{\prime}=\max \left\{d_{0}, \sup _{u \in \Omega_{1}}\|u\|\right.$, $\left.\sup _{u \in \Omega_{2}}\|u\|\right\}+1$. It follows from the definition of $\Omega_{1}$ and $\Omega_{2}$ that $M u \neq N_{\lambda} u, \lambda \in(0,1), u \in \partial \Omega$ and $Q N u \neq 0$, $u \in \partial \Omega \cap \operatorname{Ker} M$.

Define a homeomorphism $J: \operatorname{Im} Q \rightarrow \operatorname{Ker} M$ as $J\left(k e^{-t}\right)=k t$. If $d f(t, x, d)<0$ for $|d|>d_{0}$, take the homotopy

$$
H(u, \mu)=\mu u+(1-\mu) J Q N u, \quad u \in \bar{\Omega} \cap \operatorname{Ker} M, \mu \in[0,1] .
$$

For $u \in \bar{\Omega} \cap \operatorname{Ker} M$, we have $u=k t$. Then

$$
H(u, \mu)=\mu k t-(1-\mu) \frac{\sum_{i=1}^{n} \alpha_{i} \int_{\xi_{i}}^{+\infty} f(s, k s, k) d s}{\sum_{i=1}^{n} \alpha_{i} e^{-\xi_{i}}} t .
$$

Obviously, $H(u, 1) \neq 0, u \in \partial \Omega \cap \operatorname{Ker} M$. For $\mu \in[0,1), u=k t \in \partial \Omega \cap \operatorname{Ker} M$, if $H(u, \mu)=0$, we have

$$
\frac{\sum_{i=1}^{n} \alpha_{i} \int_{\xi_{i}}^{+\infty} k f(s, k s, k) d s}{\sum_{i=1}^{n} \alpha_{i} e^{-\xi_{i}}}=\frac{\mu}{1-\mu} k^{2} \geq 0 .
$$

A contradiction with $d f(t, x, d)<0,|d|>d_{0}$. If $d f(t, x, d)>0,|d|>d_{0}$, take

$$
H(u, \mu)=\mu u-(1-\mu) J Q N u, \quad u \in \bar{\Omega} \cap \operatorname{Ker} M, \mu \in[0,1],
$$

and the contradiction follows analogously. So, we obtain $H(u, \mu) \neq 0, \mu \in[0,1], u \in \partial \Omega \cap$ $\operatorname{Ker} M$.

By the homotopy of degree, we get that

$$
\begin{aligned}
\operatorname{deg}(J Q N, \Omega \cap \operatorname{Ker} M, 0) & =\operatorname{deg}(H(\cdot, 0), \Omega \cap \operatorname{Ker} M, 0) \\
& =\operatorname{deg}(H(\cdot, 1), \Omega \cap \operatorname{Ker} M, 0)=\operatorname{deg}(I, \Omega \cap \operatorname{Ker} M, 0)=1 .
\end{aligned}
$$

By Theorem 2.1, we can get that $M u=N u$ has at least one solution in $\bar{\Omega}$. The proof is completed.

\section{Example}

Let us consider the following boundary value problem at resonance

$$
\left\{\begin{array}{l}
\left(\left|u^{\prime}\right|^{-\frac{1}{2}} u^{\prime}\right)^{\prime}+\frac{e^{-4 t}}{\sqrt{1+t}} \sin \sqrt{|u|}+e^{-4 t}\left|u^{\prime}\right|^{-\frac{1}{2}} u^{\prime}+\frac{1}{4} e^{-4 t}=0, \quad 0<t<+\infty \\
u(0)=0, \quad\left|u^{\prime}(+\infty)\right|^{-\frac{1}{2}} u^{\prime}(+\infty)=\sum_{i=1}^{n} \alpha_{i}\left|u^{\prime}\left(\xi_{i}\right)\right|^{-\frac{1}{2}} u^{\prime}\left(\xi_{i}\right),
\end{array}\right.
$$

where $0<\xi_{1}<\xi_{2}<\cdots<\xi_{n}<+\infty, \alpha_{i}>0, \sum_{i=1}^{n} \alpha_{i}=1$

Corresponding to problem (1.1), we have $p=\frac{3}{2}, f(t, x, y)=\frac{e^{-4 t}}{\sqrt{1+t}} \sin \sqrt{|x|}+e^{-4 t}|y|^{-\frac{1}{2}} y+$ $\frac{1}{4} e^{-4 t}$.

Take $a(t)=\frac{e^{-4 t}}{\sqrt{1+t}}, b(t)=e^{-4 t}, c(t)=\frac{1}{4} e^{-4 t}, d_{0}=4$. By simple calculation, we can get that conditions $\left(\mathrm{H}_{1}\right)-\left(\mathrm{H}_{4}\right)$ hold. By Theorem 3.1, we obtain that problem (4.1) has at least one solution. 


\section{Competing interests}

The author declares that she has no competing interests.

\section{Author's contributions}

All results belong to WJ.

\section{Acknowledgements}

This work is supported by the National Science Foundation of China (11171088) and the Natural Science Foundation of Hebei Province (A2013208108). The author is grateful to anonymous referees for their constructive comments and suggestions, which led to the improvement of the original manuscript.

Received: 5 April 2013 Accepted: 23 August 2013 Published: 11 September 2013

\section{References}

1. Mawhin, J: Topological Degree Methods in Nonlinear Boundary Value Problems. NSFCBMS Regional Conference Series in Mathematics. Am. Math. Soc., Providence (1979)

2. Mawhin, J: Resonance problems for some non-autonomous ordinary differential equations. In: Johnson, R, Pera, MP (eds.) Stability and Bifurcation Theory for Non-Autonomous Differential Equatons. Lecture Notes in Mathematics, vol. 2065, pp. 103-184. Springer, Berlin (2013)

3. Feng, W, Webb, JRL: Solvability of $m$-point boundary value problems with nonlinear growth. J. Math. Anal. Appl. 212, 467-480 (1997)

4. Ma, R: Existence results of a m-point boundary value problem at resonance. J. Math. Anal. Appl. 294, 147-157 (2004)

5. Zhang, X, Feng, M, Ge, W: Existence result of second-order differential equations with integral boundary conditions at resonance. J. Math. Anal. Appl. 353, 311-319 (2009)

6. Du, Z, Lin, X, Ge, W: Some higher-order multi-point boundary value problem at resonance. J. Comput. Appl. Math 177, 55-65 (2005)

7. Kosmatov, N: A boundary value problem of fractional order at resonance. Electron. J. Differ. Equ. 135, 1-10 (2010)

8. Jiang, W: The existence of solutions to boundary value problems of fractional differential equations at resonance. Nonlinear Anal. TMA 74, 1987-1994 (2011)

9. Jiang, W: Solvability for a coupled system of fractional differential equations at resonance. Nonlinear Anal., Real World Appl. 13, 2285-2292 (2012)

10. Jiang, W: Solvability of $(k, n-k)$ conjugate boundary-value problems at resonance. Electron. J. Differ. Equ. 114, 1-10 (2012)

11. Ge, W, Ren, J: An extension of Mawhin's continuation theorem and its application to boundary value problems with a p-Laplacian. Nonlinear Anal. 58, 477-488 (2004)

12. Liu, Y, Li, D, Fang, M: Solvability for second-order $m$-point boundary value problems at resonance on the half-line. Electron. J. Differ. Equ. 2009, 13 (2009)

13. Liu, Y: Boundary value problem for second order differential equations on unbounded domain. Acta Anal. Funct. Appl. 4(3), 211-216 (2002) (in Chinese)

14. Agarwal, RP, O’Regan, D: Infinite Interval Problems for Differential, Difference and Integral Equations. Kluwer Academic, Dordrecht (2001)

15. Kosmatov, N: Multi-point boundary value problems on an unbounded domain at resonance. Nonlinear Anal. 68 2158-2171 (2008)

doi:10.1186/1687-2770-2013-207

Cite this article as: Jiang: Solvability for $p$-Laplacian boundary value problem at resonance on the half-line. Boundary Value Problems 2013 2013:207.

\section{Submit your manuscript to a SpringerOpen ${ }^{\ominus}$ journal and benefit from:}

- Convenient online submission

- Rigorous peer review

- Immediate publication on acceptance

- Open access: articles freely available online

- High visibility within the field

- Retaining the copyright to your article 